\title{
Structure Effect on Antioxidant Activity of Catecholamines toward Singlet Oxygen and Other Reactive Oxygen Species in vitro
}

\author{
Takako Shimizu$^{1}$, Yuji Nakanishi', Meiko Nakahara ${ }^{2}$, Naoki Wada ${ }^{1}$, Yoshihiko Moro-oka ${ }^{3}$, \\ Toru Hirano $^{4}$, Tetsuya Konishi ${ }^{5}$ and Seiichi Matsugo ${ }^{1, *}$ \\ ${ }^{1}$ School of Natural System, College of Science and Engineering, Kanazawa University, \\ Kakuma-machi, Kanazawa 920-1192, Japan \\ ${ }^{2}$ Interdisciplinary Graduate School of Medicine and Engineering, University of Yamanashi, \\ Takeda 4-3-11, Kofu, Yamanashi 400-8511, Japan \\ ${ }^{3}$ Emeritus Prof. of Tokyo Institute of Technology \\ ${ }^{4}$ Hamamatsu University School of Medicine, Photon Medical Research Center, \\ Hamamatsu, Handayama, Higashi-ku, Hamamatsu 431-3192, Japan \\ ${ }^{5}$ Faculty of Applied Life Sciences, Niigata University of Pharmacy \& Applied Life Sciences, \\ Higashijima 265-1, Akiha-ku, Niigata 956-2081, Japan
}

Received 16 November, 2009; Accepted 22 December, 2009; Published online 16 September, 2010

\begin{abstract}
Summary The reactivity of catecholamine neurotransmitters and the related metabolites were precisely investigated toward 1,1-diphenyl-2-picrylhydrazyl (DPPH) radicals and reactive oxygen species. Catecholamines reacted immediately with DPPH radicals, their reactivity being stronger than that of ascorbic acid as a reference. Superoxide scavenging activities of catecholamines determined by WST-1 and electron spin resonance (ESR) spin trapping methods were also high. Whereas tyrosine, the dopamine precursor showed no reactivity toward superoxide. The reactivity toward singlet oxygen was evaluated by observing specific photon emission from singlet oxygen. The results revealed that reactivity of catecholamines was markedly higher than that of sodium azide, and catechin as catechol reference. The reaction of catecholamines and singlet oxygen was further studied by ESR using 55-dimethyl1-pyrroline $\mathrm{N}$-oxide (DMPO) as a spin trapping reagent and rose bengal as photosensitizer. DMPO-OH signal of epinephrine was significantly small compared to other catecholamines, catechin, and 4-methylcatechol as a reference compound and was as small as that of tyrosine. The signal formation was totally dependent on singlet oxygen, and the presence of catechol compounds. These results indicated that epinephrine is the most potent singlet oxygen quencher than other catecholamines, and the secondary amino group in its alkyl side chain could play a role in unique singlet oxygen quenching property of epinephrine.
\end{abstract}

Key Words: catecholamine, neurotransmitters, ROS, chemiluminescence, singlet oxygen

\section{Introduction}

The catecholamines dopamine, norepinephrine, and epi-

\footnotetext{
*To whom correspondence should be addressed.

Tel: +81-76-264-6219 Fax: +81-76-234-4829

E-mail: matsugoh@t.kanazawa-u.ac.jp
}

nephrine function as neurotransmitters in the central nervous system and also as hormones in the peripheral endocrine system [1]. These compounds contain a characteristic catechol chromophore, as indicated in their family name, and are biosynthesized from tyrosine through an 3,4-dihydroxy-Lphenylalanine (L-DOPA). Catecholamines affect the brain regions associated with emotional activities such as hyperexcitability and depression [2]. The catechol structure is 
widely distributed in many naturally occurring antioxidants and plays a role in scavenging reactive oxygen species (ROS), typically, superoxide. Therefore, catecholamine neurotransmitters and related metabolites are also expected to react with ROS.

The brain is vulnerable to oxidative stress caused by high contents of oxidizable substrates, such as polyunsaturated fatty acid (PUFA) [3]. The brain consumes ca. 20\% of inhaled oxygen, thus, a large amount of ROS are believed to be produced under normal metabolic and physiologic states [4]. ROS produced in the brain have been associated with various neurodegenerative disorders such as Parkinson's disease, which is characterized by the dysfunction of dopaminergic neurons in the nigrostriatal system [5].

The external administration of antioxidant is usually not effective to prevent brain oxidative stress because of the presence of the blood/brain/barrier (BBB) [6]. In addition, the activity of typical antioxidant enzymes such as superoxide dismutase (SOD) is lower in the brain than in other tissues such as liver [7]. Hence, we focused our attention on the reactivity of catecholamine neurotransmitters toward ROS and radical including 1,1-diphenyl-2-picrylhydrazyl (DPPH), superoxide, and singlet oxygen (Fig. 1). Superoxide is the most abundant ROS generated under physiological conditions and it is also generated by the auto-oxidation of dopamine [8]. Singlet oxygen, on the other hand, has been paid little attention on the oxidative stress compared with other ROS, although it causes damages DNA [9], PUFA [10], and amino acid [11] at the locus of generation. It is produced by a photosensitized reaction (photodynamic<smiles>NCCc1ccc(O)c(O)c1</smiles>

dopamine<smiles>CNC[C@H](O)c1ccc(O)c(O)c1</smiles>

norepinephrine<smiles>NC[C@H](O)c1ccc(O)c(O)c1</smiles>

epinephrine<smiles>N[C@H](Cc1ccc(O)cc1)C(=O)O</smiles>

tyrosine<smiles>N[C@@H](Cc1ccc(O)c(O)c1)C(=O)O</smiles>

L-DOPA<smiles>Oc1cc(O)c2c(c1)O[C@H](c1ccc(O)c(O)c1)[C@H](O)C2</smiles>

catechin

Fig. 1. Chemical structures of catecholamine neurotransmitters and catechin. action) and also by enzyme-catalyzed reactions such as myeloperoxidase-hydrogen peroxide-chloride reaction in vivo system [12]. It also plays a role in cellular signaling and apoptosis [13-15]. Therefore, it is necessary to study the reactivity of singlet oxygen toward neurotransmitter molecules. Although several fragmented studies have been reported on the reactions of catecholamines toward ROS, we have re-evaluated the scavenging activity of a series of catecholamine neurotransmitters towards ROS, with special focus on their reactivity toward singlet oxygen.

\section{Materials and Methods}

\section{Reagents}

Dopamine hydrochloride, 3,4-dihydroxy-L-phenylalanine (L-DOPA), L-noradrenaline (norepinephrine), L-adrenaline (epinephrine), tyrosine, 1,1-diphenyl-2-picrylhydrazyl (DPPH), 4-methyl catechol, hypoxanthine, and xanthine oxidase (XOD) were purchased from Nacalai Tesque Co. (Kyoto, Japan). Ascorbic acid and rose bengal were purchased from Sigma-Aldrich Co. Japan (Tokyo, Japan). 4-[3-(4-iodophenyl)-2-(4-nitrophenyl)-2H-5-tetrazolio]-1,3benzene disulfonate sodium salt (WST-1) and 55-Dimethyl1-pyrroline N-oxide (DMPO) were purchased from Dojindo Co. (Kumamoto, Japan). (+)-Catechin hydrate was purchased from Spectrum Chemical Mfg. Corp. (Gardena, CA). Sodium azide was purchased from Wako Co. (Osaka, Japan). DLThioctic acid (lipoic acid) was purchased from Kanto chemical Co. (Tokyo, Japan). Other chemicals were of the highest grade. All reagents were used without further purification. Water was prepared using Advantec PWU-100. For the singlet oxygen photon counting method, water was prepared using Yamato Scientific WG202.

UV-Vis absorption spectra were measured by JASCO V550 UV/VIS spectrophotometer. Singlet oxygen chemiluminescence was detected by NIR-II Hamamatsu Photonics $\mathrm{KK}$, Japan.

$D P P H$ radical scavenging activity evaluated by $U V$-vis spectroscopic method

Dopamine and other test samples were dissolved in $25 \mathrm{mM}$ phosphate buffer ( $\mathrm{pH}$ 7.4) containing ethanol (final $33 \% \mathrm{v} / \mathrm{v}$ ), except epinephrine. Since the solubility of epinephrine in phosphate buffer was low, it was dissolved in a mixture of phosphate buffer and $0.1 \%$ acetic acid. Then, $0.5 \mathrm{ml}$ of $1 \mathrm{mM}$ DPPH in ethanol was added to $4.5 \mathrm{~mL}$ of catecholamine solutions of various concentrations (final ethanol $40 \% \mathrm{v} / \mathrm{v}$ ), and the solution was stirred for $30 \mathrm{~s}$. The UV-Vis absorption spectrum of this solution was obtained in the range from $350 \mathrm{~nm}$ to $700 \mathrm{~nm}$. DPPH radical scavenging activity of catecholamines was evaluated by determining the absorbance at $526.5 \mathrm{~nm}$. The reaction was also performed in $40 \%$ methanol/phosphate buffer ( $\mathrm{pH} 7.4)$, and the DPPH 
radical scavenging activity was calculated according to equation 1 (eq. 1). In the equation, $A$ and $B$ indicate DPPH absorbance in the absence and presence of the test sample, respectively.

DPPH radical scavenging activity $(\%)=100 \times(A-B) / A$

DPPH radical scavenging activity evaluated by ESR method

The test samples were dissolved in $25 \mathrm{mM}$ phosphate buffer ( $\mathrm{pH} 7.4$ ) containing methanol (final $37.5 \% \mathrm{v} / \mathrm{v}$ ), except epinephrine. Epinephrine was dissolved in a mixture of $0.4 \%$ acetic acid and phosphate buffer as described above. Then, $40 \mu \mathrm{l}$ of $1 \mathrm{mM}$ DPPH in methanol was added to $160 \mu \mathrm{l}$ of test samples of defined concentrations (final methanol $50 \% \mathrm{v} / \mathrm{v}$ ), and the solution was stirred for $30 \mathrm{~s}$. Electron spin resonance (ESR) spectra of the solution were obtained after $90 \mathrm{~s}$, and the DPPH radical scavenging activity was evaluated on the basis of the peak height of DPPH radical determined after normalization with the Mn reference peak. The conditions used for ESR measurements (JEOL JES TE200) were as follows: temperature, $22^{\circ} \mathrm{C}$; frequency, $9.04 \mathrm{GHz}$; power, $8.00 \mathrm{~mW}$; field, $341.0 \pm 10 \mathrm{mT}$; sweep time, $0.5 \mathrm{~min}$; field modulation, $0.2 \mathrm{mT}$; and amplitude, 100; and time constant, 0.03 .

Superoxide scavenging activity determined by WST-1 method The test samples of various concentrations were dissolved

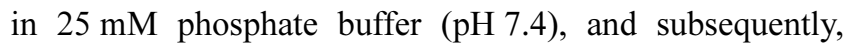
$0.1 \mathrm{U} / \mathrm{ml}$ XOD was added to the reaction mixture (total volume: $3 \mathrm{ml}$ ) containing $333 \mathrm{mM}$ WST-1, $30 \mu \mathrm{M}$ hypoxanthine, and phosphate buffer. Epinephrine was dissolved $1 \%$ acetic acid $(60 \mu \mathrm{l})$ instead of phosphate buffer as described above. The reaction was performed at room temperature and the absorbance at $432 \mathrm{~nm}$ was determined at $1 \mathrm{~min}$ after the addition of XOD. Superoxide scavenging activity was evaluated by the residual amount of WST-1 as in the following equation (eq. 2). The $A$ and $B$ indicate WST-1 absorbance in the absence and presence of the test sample, respectively.

Superoxide scavenging activity $(\%)=100 \times(A-B) / \mathrm{A}$

Superoxide scavenging activity evaluated by ESR spin trapping method

Test samples of defined concentrations were dissolved

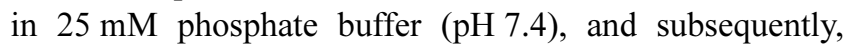
$0.1 \mathrm{U} / \mathrm{ml}$ XOD was added to the reaction mixture (total volume: $300 \mu \mathrm{l}$ ) containing $300 \mathrm{mM}$ DMPO, $533 \mu \mathrm{M}$ hypoxanthine, and phosphate buffer. Epinephrine was dissolved in $1 \%$ acetic acid $(30 \mu \mathrm{l})$ and used as described above. The ESR spectra were obtained at $50 \mathrm{~s}$ after the addition of XOD, and the superoxide scavenging activity was evaluated from the peak height of DMPO-OOH signal (the superoxide DMPO adduct) after normalization with the Mn reference peak [16]. The conditions used for ESR measurement were the same as mentioned before.

Singlet oxygen scavenging activity evaluated by photon counting method

To evaluate the singlet oxygen scavenging activity of catecholamines, we dissolved the test samples and rose bengal (photosensitizer) in deionized water at the concentrations of $1 \mathrm{mM}$ and $30 \mu \mathrm{M}$, respectively. The reaction mixture containing test sample and $3 \mathrm{ml}$ of rose bengal was dispensed in a quartz cuvette and irradiated by YAG laser at $532 \mathrm{~nm}(30 \mathrm{~Hz}, 40 \mathrm{~mW})$ with a silicon filter $(1100 \mathrm{~nm}$ cut-off). Photon emission from singlet oxygen was detected at $1268 \mathrm{~nm}$ using a highly sensitive near infrared (NIR) spectrophotometer [17]. Data correction was achieved by keeping a $5 \mu$ s time-lag after every pulse irradiation to avoid the effect of strong artificial background fluorescence from rose bengal. To evaluate the dose dependant activity of catecholamines, we added $50 \mu \mathrm{l}$ of test sample (up to $250 \mu \mathrm{l}$ ) to the reaction mixture in a cuvette, and determined the absorbance of the solution at each step. Singlet oxygen scavenging activity was evaluated based on the signal intensity of the peaks.

Reaction of catecholamines with singlet oxygen in the presence of DMPO

The reaction mixture containing $250 \mu \mathrm{M}$ test sample, $225 \mu \mathrm{M}$ DMPO, and $24.5 \mu \mathrm{M}$ rose bengal was prepared in $25 \mathrm{mM}$ phosphate buffer ( $\mathrm{pH}$ 7.4) (total volume: $200 \mu \mathrm{l}$ ). The dose dependent activities of sodium azide and lipoic acid were determined in the presence of catechin $(250 \mu \mathrm{M})$ or dopamine $(250 \mu \mathrm{M})$, respectively. The reaction solution was placed at $12 \mathrm{~cm}$ from the halogen lamp $(650 \mathrm{~W})$ and irradiated by visible light for $1 \mathrm{~min}$. After $100 \mathrm{~s}$, the ESR spectrum of the mixture was obtained (JES-FR30EX).

\section{Statistical analyses}

All data presented in this study were the average of at least three times experiments and expressed as mean \pm SD values. Data were analyzed by Student's $t$ test.

\section{Results}

\section{DPPH radical scavenging activity}

The DPPH radical scavenging activity of catecholamines dissolved in aqueous ethanol revealed that the absorption bands of DPPH radical at $526.5 \mathrm{~nm}$ decreased after the addition of catecholamines in a concentration dependent manner. The relative DPPH radical quenching ability of catecholamines and their $\mathrm{IC}_{30}$ values (the concentrations 
Table 1. DPPH radical scavenging activity determined by UV-vis spectroscopy

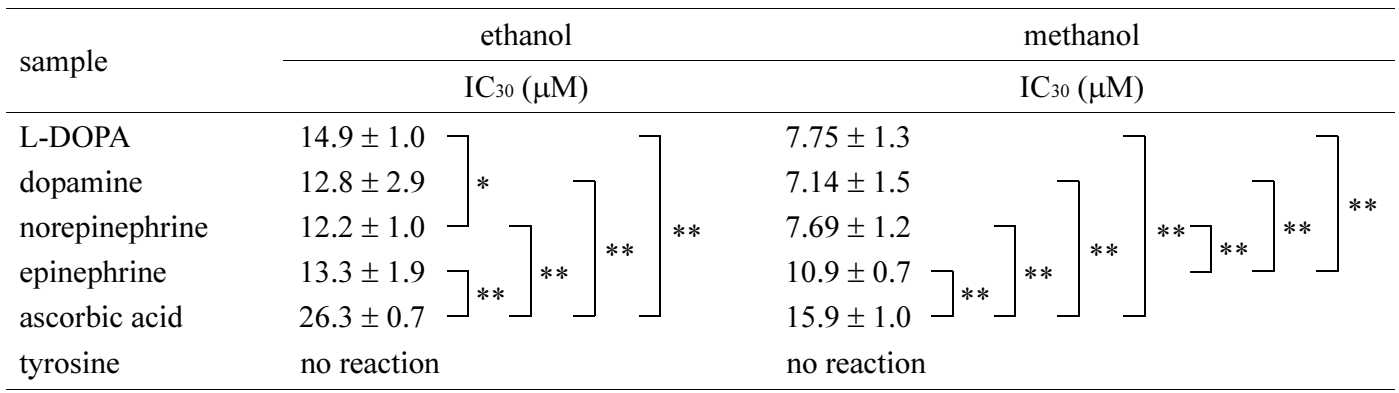

${ }^{*} p<0.05,{ }^{*} p<0.01$ compared with the indicated group using student $t$ test.

Table 2. DPPH radical scavenging activity determined by ESR spectroscopy

\begin{tabular}{ll}
\hline \multirow{2}{*}{ sample } & \multicolumn{2}{c}{ methanol } \\
\cline { 2 - 3 } & \multicolumn{2}{c}{$\mathrm{IC}_{50}(\mu \mathrm{M})$} \\
\hline L-DOPA & $7.51 \pm 1.6$ \\
dopamine & $6.33 \pm 0.8$ \\
norepinephrine & $7.10 \pm 1.1$ \\
epinephrine & $11.8 \pm 1.0$ \\
ascorbic acid & $15.9 \pm 2.3,7 * 7 * 7 * 7 * 7 * 7 * 7 *] *$ \\
tyrosine & no reaction
\end{tabular}

${ }^{*} p<0.05,{ }^{* *} p<0.01$ compared with the indicated group using student $t$ test.

required for scavenging $30 \%$ of DPPH radicals) are summarized in Table 1 . The reactivity of catecholamines in aqueous ethanol was in the following order: norepinephrine $>$ dopamine $>$ epinephrine $>$ L-DOPA $>$ ascorbic acid. Tyrosine did not scavenge DPPH radical in the same concentration range as that of catecholamines.

The DPPH scavenging activity was also determined in aqueous methanol. The $\mathrm{IC}_{30}$ values obtained in aqueous methanol were significantly smaller than those obtained in aqueous ethanol. However, in both solutions, all the examined catecholamines showed stronger scavenging activity than ascorbic acid.

The DPPH radical scavenging activity of catecholamines was further determined by ESR. Since this method afforded a clear dose response up to $80 \%$ inhibition level, the scavenging activity of catecholamines was compared on the basis of the $\mathrm{IC}_{50}$ values (the concentration scavenging $50 \%$ of the original DPPH); these are presented in Table 2. The DPPH radical scavenging activities in aqueous methanol system obtained by ESR were similar to those determined by UV-Vis spectroscopy as described above. The scavenging activity of epinephrine was lower than that of other catecholamines, as determined by both these methods. The

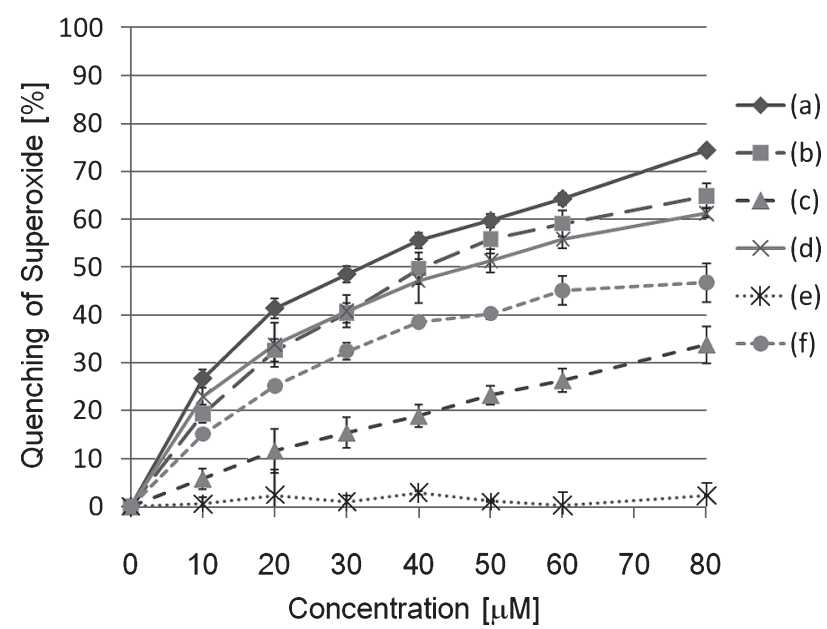

Fig. 2. Superoxide scavenging activity determined by WST-1 method. The decrease in the absorbance at $432 \mathrm{~nm}$ due to superoxide-induced formazan formation by WST-1 reduction was measured by UV-Vis spectrometer in the presence and absence of catecholamine. Superoxide was generated in the hypoxanthine-xanthine oxidase system. (a) dopamine, (b) norepinephrine, (c) epinephrine, (d) L-DOPA, (e) tyrosine, (f) ascorbic acid.

reactivity of catecholamines was in the following order: dopamine $>$ norepinephrine $>$ L-DOPA $>$ epinephrine $>$ ascorbic acid. Tyrosine did not affect the peak height of the DPPH radical signal.

\section{Superoxide scavenging activity}

Superoxide scavenging activity of catecholamines was determined by the WST-1 method, using tyrosine and ascorbic acid as reference agents. The dose dependant reactivity of catecholamines is shown in Fig. 2, and the $\mathrm{IC}_{30}$ values obtained from the reaction profile are summarized in Table 3. The superoxide scavenging activity was in the following order: dopamine $>$ L-DOPA $>$ norepinephrine $>$ ascorbic acid $>$ epinephrine. Tyrosine showed no reactivity 
Table 3. Superoxide scavenging activity determined by WST-1 reduction

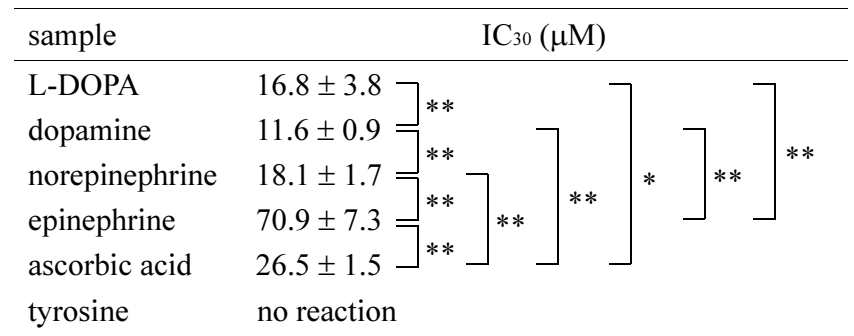

${ }^{*} p<0.05,{ }^{* *} p<0.01$ compared with the indicated group using student $t$ test.
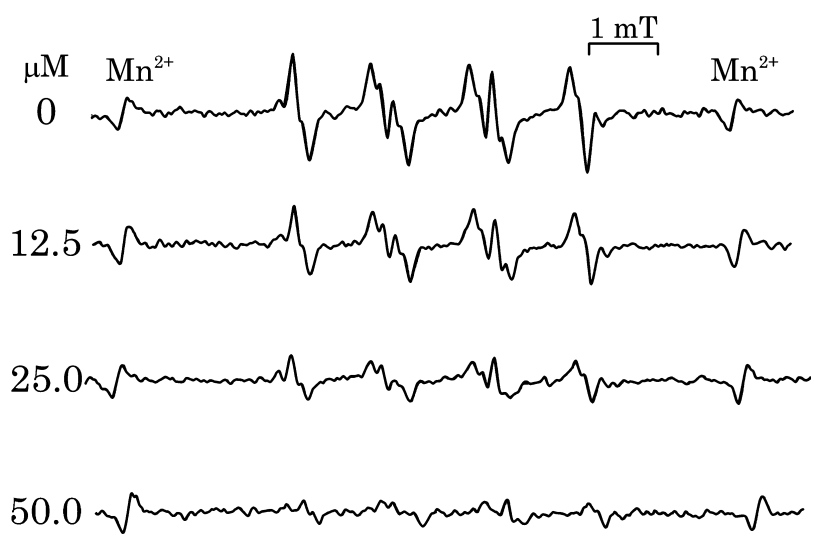

Fig. 3. Dopamine dependent decrease of DMPO-OOH signal. Superoxide was determined by ESR spin trapping method using DMPO as the spin trap reagent and hypoxanthinexanthine oxidase system as superoxide generator. Precise ESR measurement condition is given in the experimental section.

toward superoxide.

The superoxide scavenging activity was further evaluated by ESR using spin trapping method. The typical ESR spectra of DMPO-OOH signal in the presence of different concentrations of dopamine are shown in Fig. 3. The DMPO-OOH signal intensity decreased in a concentration dependent manner.

The $\mathrm{IC}_{50}$ values of different catecholamines determined by the ESR method are listed in Table 4. The relative activities of catecholamines determined by ESR method were similar to those obtained by WST-1 method. The scavenging activity of dopamine, norepinephrine, and LDOPA was higher than that of ascorbic acid, while the scavenging activity of epinephrine was lower than that of other catecholamines and ascorbic acid. The relative activity was in the following order: dopamine $>$ L-DOPA $>$ norepinephrine $>$ ascorbic acid $>$ epinephrine.
Table 4. Superoxide scavenging activity determined by ESR using DMPO

\begin{tabular}{|c|c|c|c|c|c|c|c|}
\hline sample & \multicolumn{7}{|c|}{$\mathrm{IC}_{50}(\mu \mathrm{M})$} \\
\hline L-DOPA & $24.3 \pm 1.1$ & & & & & & \\
\hline dopamine & $14.6 \pm 2.3=$ & $* *$ & & & & & \\
\hline norepinephrine & $25.5 \pm 4.3=$ & & & & $* *$ & $* *$ & $* *$ \\
\hline epinephrine & $89.7 \pm 4.8=$ & $* *$ & $* *$ & $* *$ & & & \\
\hline ascorbic acid & $41.2 \pm 1.6$ & $* *$ & & & & & \\
\hline tyrosine & no reaction & & & & & & \\
\hline
\end{tabular}

${ }^{*} p<0.05,{ }^{*} p<0.01$ compared with the indicated group using student $t$ test.

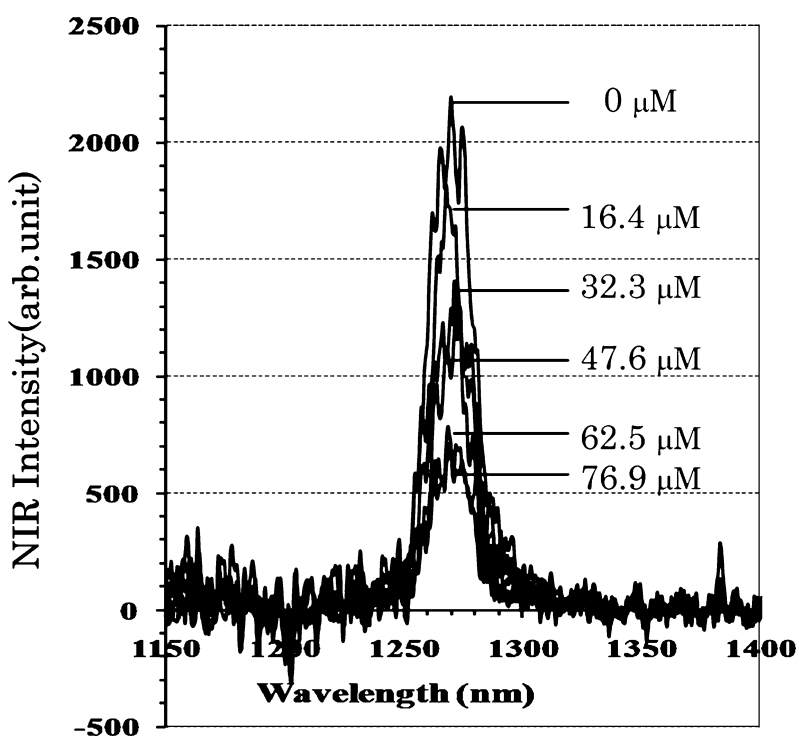

Fig. 4. Singlet oxygen quenching activity of dopamine determined by photon counting method. Rose bengal was used as a photosensitizer to generate singlet oxygen. Photon emission was determined by photon counter after laser irradiation at $532 \mathrm{~nm}$ in the presence and absence of test sample. Precise reaction condition is given experimental section.

\section{Singlet oxygen quenching activity}

When the singlet oxygen is discharged to the ground state oxygen, photon emission is observed at approximately $1268 \mathrm{~nm}$ [18]. Therefore, the photon counting of this emission is a direct evidence of singlet oxygen generation. In the present study, we determined the singlet oxygen scavenging potential of catecholamines by observing this emission using a highly sensitive NIR detection system.

The peak height of the emission spectra of singlet oxygen decreased in the presence of dopamine in a concentration dependent manner as shown in Fig. 4. The dose dependence of the quenching profile shown in Fig. 5 revealed that both 


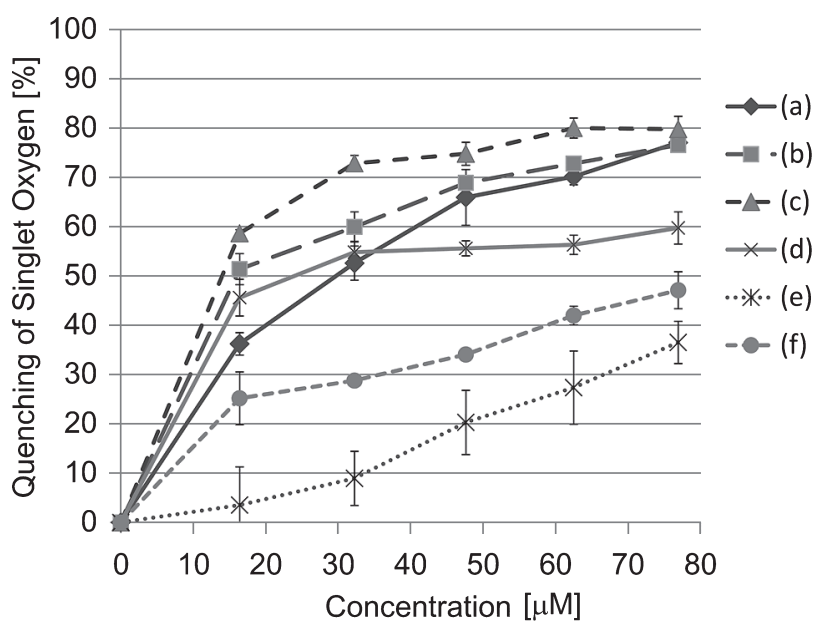

Fig. 5. Dose effect of catecholamines on singlet oxygen quenching reaction. Singlet oxygen quenching activity was measured as in Fig. 4. Precise reaction condition is given experimental section. (a) dopamine, (b) norepinephrine, (c) epinephrine, (d) L-DOPA, (e) sodium azide, (f) catechin.

epinephrine and norepinephrine have stronger quenching ability than dopamine and L-DOPA. The $\mathrm{IC}_{50}$ values of test samples are summarized in Table 5. The scavenging activities of catecholamines were significantly higher than those of catechin and sodium azide as typical singlet oxygen quencher. The reactivity was in the following order: epinephrine $>$ norepinephrine $>$ L-DOPA $>$ dopamine $>>$ catechin $>$ sodium azide.

The reactivity of catecholamines to singlet oxygen was further examined by the ESR spin trapping method using DMPO, according to a previously reported [19]. Similar to the findings of this study, we detected the characteristic DMPO-OH signal after irradiation in the presence of catecholamines or catechin (Fig. 6). Simple irradiation of rose bengal without catecholamines or catechin did not reveal any significant radical trapped by DMPO signal. Irradiation of catecholamines or catechin in the absence of rose bengal did not produce any signals. Almost same signal

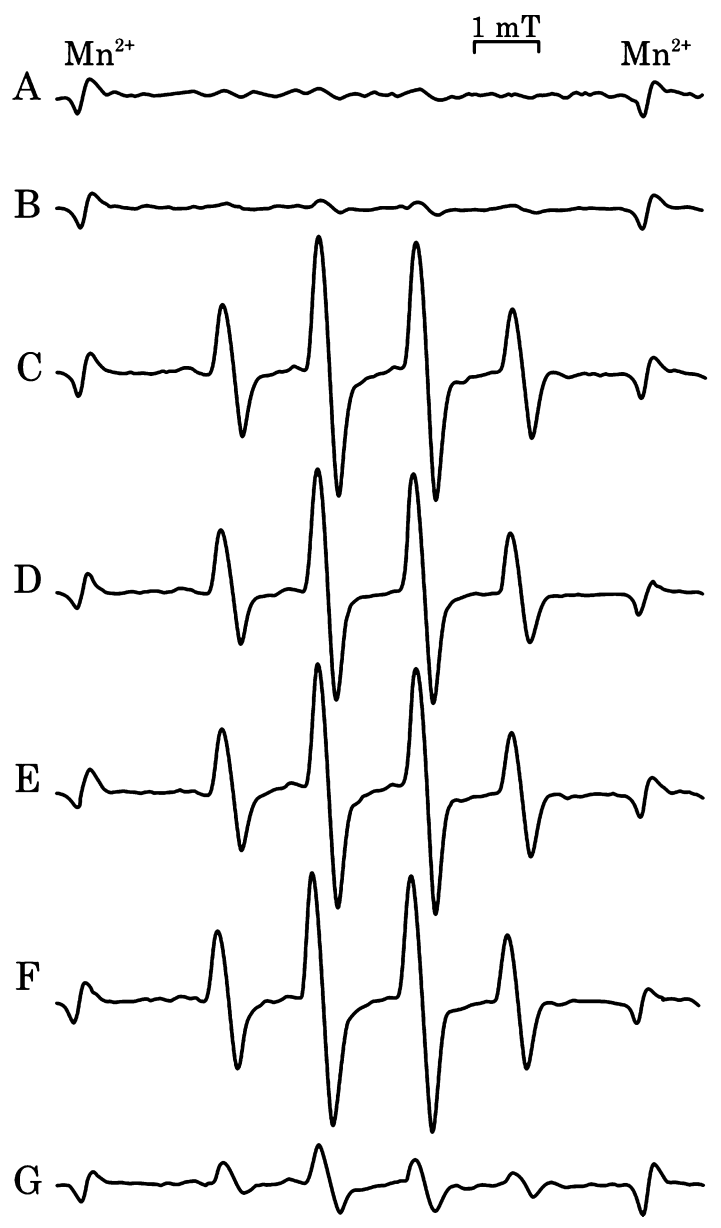

Fig. 6. Singlet oxygen scavenging activity of catecholamines evaluated by ESR spin trapping method. The reaction mixture containing catecholamines $(250 \mu \mathrm{M})$, rose bengal, and DMPO was irradiated for $1 \mathrm{~min}$, ESR spectra were measured at $100 \mathrm{~s}$ after the irradiation. Precise reaction condition is given experimental section. (A) none, (B) sodium azide, (C) catechin, (D) dopamine, (E) norepinephrine, (F) L-DOPA, (G) epinephrine.

Table 5. Singlet oxygen quenching activity evaluated by $1268 \mathrm{~nm}$ photon emission

\begin{tabular}{|c|c|c|c|c|c|c|c|c|c|c|}
\hline sample & \multicolumn{10}{|c|}{$\mathrm{IC}_{50}(\mu \mathrm{M})$} \\
\hline L-DOPA & $23.9 \pm 5.1$ & & & - & & - & - & & & \\
\hline dopamine & $29.6 \pm 3.2$ & & & & & & & $* *$ & & \\
\hline norepinephrine & $15.9 \pm 1.0$ & & & & $* *$ & $* *$ & $=$ & & & $* *$ \\
\hline epinephrine & $14.0 \pm 0.2$ & $* *$ & $* *$ & NA & & & & & $* *$ & T \\
\hline sodium azide & $181 \pm 15$ & $* *$ & & & & $* *$ & & $* *$ & & \\
\hline catechin & $140 \pm 12$ & ]* & & & & & - & - & & \\
\hline
\end{tabular}

${ }^{* *} p<0.01$ compared with the indicated group using student $t$ test. 
Table 6. Catechol dependent DMPO-OH formation in the hotodynamic singlet oxygen generating system

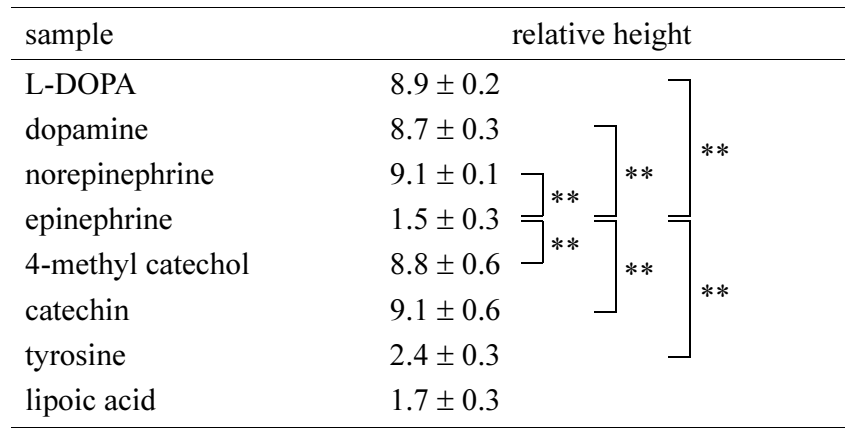

${ }^{*} p<0.05,{ }^{* *} p<0.01$ compared with the indicated group using student $t$ test. DMPO-OH was determined after the irradiation of reaction mixture containing test sample, rose bengal as photosensitizer and DMPO. Precise experimental condition was given in the experimental section.

intensity was observed for all test samples with catechol structure including 4-methyl catechol, except that the signal intensity was small in the case of epinephrine (Fig. 6, Table 6). Sodium azide inhibited this catechol dependent signal in a concentration dependent manner, thereby implying that DMPO-OH formation was dependant on the presence of singlet oxygen and that $\mathrm{OH}$-adduct formation resulted from the reaction of singlet oxygen and catecholamines (Fig. 7). Further, DMPO-OH signal formation was reduced in the presence of lipoic acid, but it was not completely inhibited even at high concentrations (up to $1.25 \mathrm{mM}$ ) (Fig. 8).

\section{Discussion}

The results in the present study revealed that catecholamine neurotransmitters were highly reactive to ROS and radical species. The DPPH and superoxide scavenging activities of catecholamines were higher than those of ascorbic acid and comparable to those of catechin, a flavan having a catechol B-ring [20], although the reactivity of epinephrine was rather weaker than those of ascorbic acid. Further, tyrosine, which has only 1 phenolic $\mathrm{OH}$ group, did not exhibit superoxide scavenging activity (Tables 3 and 4). Ohkubo et al. [21] performed laser flash photolysis and found that cumyl peroxide reacts with neurotransmitters (dopamine, norepinephrine, and epinephrine) by abstracting the hydrogen from the catechol OH group. The energy difference ( $D$ нт) between the original phenols and the resultant phenoxyl radicals was several times lesser than that in monophenols such as serotonin and tyrosine, thus, the rate of oxidation of catecholamines was at least 10 times faster than that of monophenols. This finding is consistent with our present results although the ROS is not peroxyl radical but superoxide anion radical in our present study.

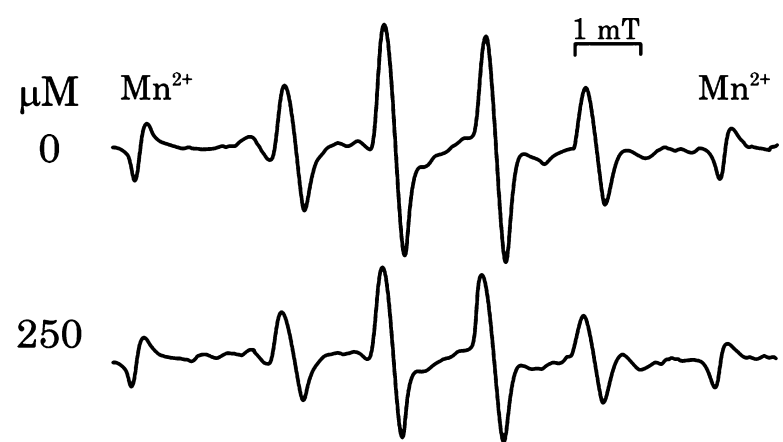

500
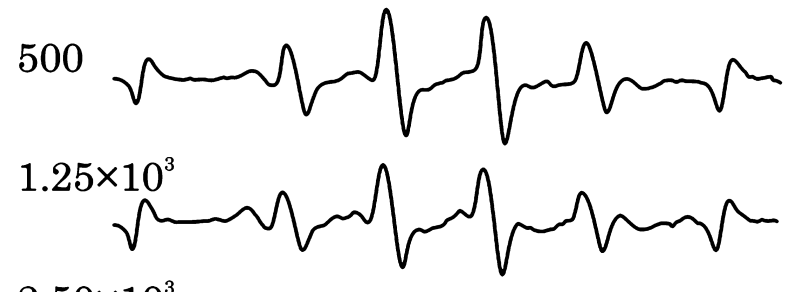

$2.50 \times 10^{3}$

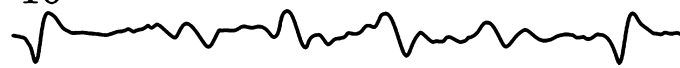

Fig. 7. Effect of sodium azide on the catechin dependent DMPO-OH formation. DMPO-OH formation was measured as in Fig. 6 at various sodium azide concentrations.

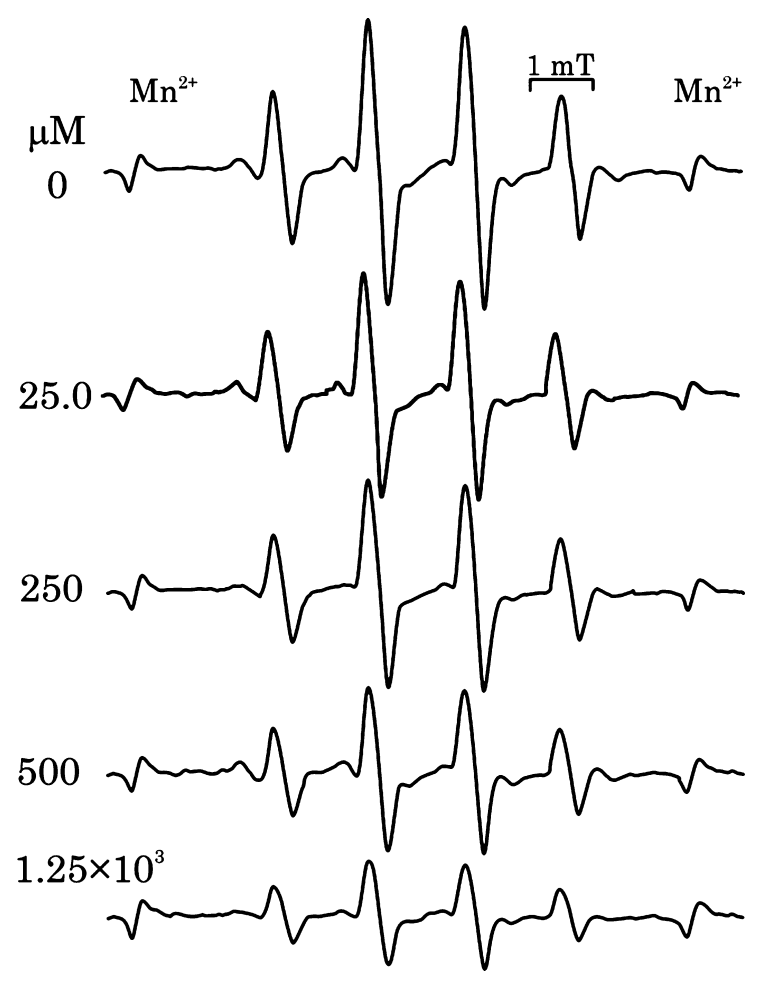

Fig. 8. Effect of lipoic acid on the dopamine dependant DMPO$\mathrm{OH}$ formation. DMPO-OH formation was measured as in Fig. 6 at various lipoic acid concentrations. 
Interestingly, we noted that the reaction of catecholamines with DPPH showed a marked solvent effect. The DPPH scavenging activities (estimated on the basis of IC values) of catecholamines determined in aqueous methanol were comparable between UV-Vis spectroscopy and ESR methods, although there was a small difference among the catecholamines. However, the $\mathrm{IC}_{30}$ values in aqueous ethanol were markedly different from those in aqueous methanol (Tables 1 and 2). When the relative scavenging activities of catecholamines were compared with those of ascorbic acid, which was used as the reference scavenging agent, the activities of catecholamines were found to be similar in both the solvents. Therefore, the DPPH assay is only adaptable for the relative comparison of free radical scavenging activities of a series of test samples, and a common reference is required for accurate evaluation of scavenging activity. Further, our results indicated that the superoxide scavenging activity of epinephrine was lower than that of other catecholamines. The same tendency was observed for DPPH radical scavenging activity. Among all the catecholamines used in this study, dopamine showed the highest DPPH and superoxide scavenging activities in all assays.

Further, the present study revealed the unique reactivity of catecholamines toward singlet oxygen, whose reactivity was different from that of other ROS. It is not a free radical but a strong oxidant since it has unique electronic configuration and $94.1 \mathrm{~kJ} / \mathrm{mol}$ higher energy than the ground state oxygen [22]. Singlet oxygen can cause damage in a wide range of biologically important molecules [23]. The involvement of singlet oxygen in a reaction is usually indirectly evaluated on the basis of the presence of characteristic products, such as "ene"-type products, or by using specific quenchers, such as 1,4-diazabicyclo[2.2.2] octane (DABCO) or sodium azide $[24,25]$. In the present experiment, the reaction of singlet oxygen with catecholamines and catechin were directly examined by the photon counting method. The obtained results revealed that the singlet oxygen quenching activity of catecholamines was several times stronger than that of catechin and sodium azide (Fig. 5).

The quenching of singlet oxygen proceeds via chemical and physical processes. Sodium azide quenches singlet oxygen mainly by the physical process [26]. It has been reported that catechin, the green tea polyphenol, quenches singlet oxygen mainly by the physical process and that the catechol structure at B-ring is responsible for the physical quenching process $[27,28]$. It was also known that singlet oxygen interacts with certain amino acids such as tryptophan, histidine, and tyrosine mainly by the chemical process. For example, tyrosine and tyrosine derivatives that bear electrondonating groups on their aromatic rings readily react with singlet oxygen via the chemical process [29, 30]. In our study, the reactivity of catecholamines toward singlet oxygen, which was determined by evaluating the quenching activity corresponding to the $1268 \mathrm{~nm}$ emission peak, was considerably higher than that of catechin and sodium azide. Among the catecholamines examined, norepinephrine and epinephrine exhibited stronger quenching activities than dopamine and L-DOPA did (Table 5). Therefore it was expected not only the catechol structure but other factor such as alkyl side chain property are involved in the singlet oxygen quenching probably via both physical and chemical processes.

In the present study, the reaction of singlet oxygen and catecholamines was further examined by ESR using DMPO as the spin trapping reagent. It has been reported that the hydroxyl radical adduct of DMPO (DMPO-OH) is formed in a photosensitized singlet oxygen generating system in the presence of phenolic antioxidants or reduced glutathione, thereby indicating that the hydrogen donating property of antioxidants is essential for the formation of DMPO-OH $[31,32]$. Indeed, in our experiment, catecholamines and catechin produced large DMPO-OH signals, however lipoic acid, which is a strong antioxidant but is not a hydrogen donating molecule, formed only a weak DMPO-OH signal (Table 6). Further, catechin dependent DMPO-OH formation was inhibited by sodium azide as singlet oxygen quencher in a concentration dependent manner (Fig. 7). The DMPO-OH formation was therefore found to be primarily dependent on the generation of singlet oxygen, as reported previously [19, 31, 32]. Tyrosine did not give rise to significant signal, although it has the same alkyl side chain structure as L-DOPA but does not have catechol structure. However, 4-methyl catechol, which is used as the reference for catecholamine, formed as large DMPO-OH signal as that of catecholamines (Table 6). Therefore, a catechol structure is the primary requisite for DMPO-OH formation, and the contribution of alkyl side chain to this process may not be large. The interesting finding is that the DMPO-OH signal of epinephrine was significantly small compared to other catecholamines including norepinephrine, and the signal level was as small as that of tyrosine even though it has catechol structure.

Nishizawa et al. [31] reported the mechanism of the DMPO-OH formation that the DMPO-singlet oxygen adducts primarily generated is transformed into $\mathrm{DMPO}-\mathrm{OH}$ in the presence of hydrogen donating antioxidant, and they also suggested that the free hydroxyl radical produced by catechol oxidation is involved in DMPO-OH formation. To evaluate the contribution of free hydroxyl radical in the DMPO-OH formation, we studied the effect of lipoic acid on the reaction, because lipoic acid and dihydrolipoic acid are known to effectively scavenge hydroxyl radicals generated from metal free system [33, 34]. Increasing concentrations of lipoic acid inhibited catecholamine dependent DMPO$\mathrm{OH}$ formation, but this inhibitory effect was not very 
remarkable (Fig. 8). Therefore, the free hydroxyl radicals might not be the major source of DMPO-OH. Moreover, epinephrine inhibited the dopamine dependant DMPO-OH signal in a concentration dependent manner (data not shown). Taken these discussions together, DMPO-OH signal suppressing effect of epinephrine might be due to its higher singlet oxygen scavenging activity. This is supported from the data in Table 5 in that epinephrine showed the highest reactivity toward singlet oxygen among the catecholamines. The only structural difference between epinephrine and norepinephrine is the alkyl side chain, the former has a secondary amino group while the latter has the primary amino group. Therefore, it can be presumed that owing to the presence of the secondary amino group, epinephrine exhibits higher activity toward singlet oxygen as compared to other catecholamines, which has a primary amino group.

The present study showed the high reactivity of catechol neurotransmitters (dopamine, norepinephrine, or epinephrine) and their precursor (L-DOPA) toward ROS. Therefore, under oxidative stress conditions, catecholamine neurotransmitters will readily react with ROS in the brain and oxidize dopamine to semiquinone, quinone, and dopaminochrome, which exert neurotoxic effects [35-40]. Although the critical role of singlet oxygen in brain disorders has not been elucidated yet, the results of this study suggest that further investigation to understand the significant contribution of singlet oxygen-catecholamine neurotransmitter reactions in brain disorders is warranted in the future.

\section{References}

[1] Hauser, S.L. and Longo, D.L.: Part IV Clinical Pharmacology, in Harrison's Principles of Internal Medicine 15th, eds. By Braunwald, E., Fauci, A.S., Kasper, D.L., and Jameson, J.L., McGraw-Hill Professional Publishing, New York, pp. 427-457, 2006.

[2] McQueen, J.K.: Biochemistry of transmitter molecules, in Transmitter molecules in the brain, eds. By Flink, G., McQueen, J.K., Harmar, A.J., Arbuthnott, G.W., Mitchell, R., and Christie, J.E., Springer, Berlin, pp. 10-16, 1987.

[3] Liu, X., Yamada, N., Maruyama, W., and Osawa, T.: Formation of dopamine adducts derived from brain polyunsaturated fatty acids: mechanism for Parkinson disease. $J$. Biol. Chem., 283, 34887-34895, 2008.

[4] Floyd, R.A.: Antioxidants, oxidative stress, and degenerative neurological disorders. Proc. Soc. Exp. Biol. Med., 222, 236245, 1999.

[5] Serra, P.A., Esposito, G., Enrico, P., Mura, M.A., Migheli, R., Delogu, M.R., Miele, M., Desole, M.S., Grella, G., and Miele, E.: Manganese increases L-DOPA auto-oxidation in the striatum of the freely moving rat: potential implications to L-DOPA long-term therapy of Parkinson's disease. Br. J. Pharmacol., 130, 937-945, 2000.

[6] Gilgun-Sherki, Y., Melamed, E., and Offen, D.: Oxidative stress induced-neurodegenerative diseases: the need for antioxidants that penetrate the blood brain barrier. Neuropharmacology, 40, 959-975, 2001.

[7] Marklund, S.L., Westman, N.G., Lundgren, E., and Roos, G.: Copper- and zinc-containing superoxide dismutase, manganese-containing superoxide dismutase, catalase, and glutathione peroxidase in normal and neoplastic human cell lines and normal human tissues. Cancer Res., 42, 19551961, 1982.

[8] Graham, D.G.: Oxidative pathways for catecholamines in the genesis of neuromelanin and cytotoxic quinones. Mol. Pharmacol., 14, 633-643, 1978.

[9] Cadet, J., Douki, T., and Ravanat, J.L.: Oxidatively generated damage to the guanine moiety of DNA: mechanistic aspects and formation in cells. Acc. Chem. Res., 41, 1075-1083, 2008.

[10] Schafer, F.Q. and Buettner, G.R.: Singlet oxygen toxicity is cell line-dependent: a study of lipid peroxidation in nine leukemia cell lines. Photochem. Photobiol., 70, 858-867, 1999.

[11] Davies, M.J. and Truscott, R.J.: Photo-oxidation of proteins and its role in cataractogenesis. J. Photochem. Photobiol. B., 63, 114-125, 2001.

[12] Kiryu, C., Makiuchi, M., Miyazaki, J., Fujinaga, T., and Kakinuma, K.: Physiological production of singlet molecular oxygen in the myeloperoxidase-H2O2-chloride system. FEBS Lett., 443, 154-158, 1999.

[13] Reiter, R.J.: Oxidative damage in the central nervous system: protection by melatonin. Prog. Neurobiol., 56, 359-384, 1998.

[14] Klotz, L.O.: Oxidant-induced signaling: effects of peroxynitrite and singlet oxygen. Biol. Chem., 383, 443-456, 2002.

[15] Chan, W.H., Yu, J.S., and Yang, S.D.: Apoptotic signaling cascade in photosensitized human epidermal carcinoma A431 cells: involvement of singlet oxygen, c-Jun N-terminal kinase, caspase-3 and p21-activated kinase 2. Biochem. J., 351, 221-232, 2000.

[16] Clément, J.L., Ferré, N., Siri, D., Karoui, H., Rockenbauer, A., and Tordo, P.: Assignment of the EPR spectrum of 5,5dimethyl-1-pyrroline N-oxide (DMPO) superoxide spin adduct. J. Org. Chem., 70, 1198-1203, 2005.

[17] Hirano, T., Kohno, E., Gohto, Y., and Obama, A.: Singlet oxygen generation due to ICG irradiation. Photomedicine and Photobiology, 28, 15-16, 2006.

[18] Arakane, K., Ryu, A., Takarada, K., Masunaga, T., Shinmoto, K., Kobayashi, R., Mashiko, S., Nagano, T., and Hirobe, M.: Measurement of $1268 \mathrm{~nm}$ emission for comparison of singlet oxygen (1 delta g) production efficiency of various dyes. Chem. Pharm. Bull (Tokyo), 44, 1-4, 1996.

[19] Ueda, J., Takeshita, K., Matsumoto, S., Yazaki, K., Kawaguchi, M., and Ozawa, T.: Singlet oxygen-mediated hydroxyl radical production in the presence of phenols: whether DMPO-*OH formation really indicates production of *OH? Photochem. Photobiol., 77, 165-170, 2003.

[20] Rahman, M.M., Ichiyanagi, T., Komiyama, T., Hatano, Y., and Konishi, T.: Superoxide radical and peroxynitritescavenging activity of anthocyanins; structure-activity rela- 
tionship and their synergism. Free Radic. Res., 40, 9931002, 2006.

[21] Ohkubo, K., Moro-oka, Y., and Fukuzumi, S.: Hydrogen abstraction from neurotransmitters by active oxygen species facilitated by intramolecular hydrogen bonding in the radical intermediates. Org. Biomol. Chem., 4, 999-1001, 2006.

[22] Straight, R.C. and Spikes, J.D.: Photosensitized oxidation of biomolecules. In Singlet Oxygen vol. 4, ed. By Frier, A.A., CRC Press, Florida, pp. 91-143, 1985.

[23] Davies, M.J.: Singlet oxygen-mediated damage to proteins and its consequences. Biochem. Biophys. Res. Commun., 305, 761-770, 2003.

[24] Schaefer, M. and Hardeland, R.: The melatonin metabolite $\mathrm{N}$-acetyl-5-methoxykynuramine is a potent singlet oxygen scavenger. J. Pineal. Res., 46, 49-52, 2009.

[25] Baier, J., Maisch, T., Maier, M., Landthaler, M., and Bäumler, W.: Direct detection of singlet oxygen generated by UVA irradiation in human cells and skin. J. Invest. Dermatol., 127, 1498-1506, 2007.

[26] Hall, R.D. and Chignell, C.F.: Steady-state near-infrared detection of singlet molecular oxygen: a Stern-Volmer quenching experiment with sodium azide. Photochem. Photobiol., 45, 459-464, 1987.

[27] Mukai, K., Nagai, S., and Ohara, K.: Kinetic study of the quenching reaction of singlet oxygen by tea catechins in ethanol solution. Free Radic. Biol. Med., 39, 752-761, 2005.

[28] Nagai, S., Ohara, K., and Mukai, K.: Kinetic study of the quenching reaction of singlet oxygen by flavonoids in ethanol solution. J. Phys. Chem. B., 109, 4234-4240, 2005.

[29] Michaeli, A. and Feitelson, J.: Reactivity of singlet oxygen toward amino acids and peptides. Photochem. Photobiol., 59, 284-289, 1994.

[30] Wright, A., Bubb, W.A., Hawkins, C.L., and Davies, M.J.: Singlet oxygen-mediated protein oxidation: evidence for the formation of reactive side chain peroxides on tyrosine residues. Photochem. Photobiol., 76, 35-46, 2002.

[31] Nishizawa, C., Takeshita, K., Ueda, J., Mizuno, M., Suzuki, K.T., and Ozawa, T.: Hydroxyl radical generation caused by the reaction of singlet oxygen with a spin trap, DMPO, increases significantly in the presence of biological reductants. Free Radic. Res., 38, 385-392, 2004.

[32] Bilski, P., Reszka, K., Bilska, M., and Chignell, C.F.: Oxidation of the spintrap 5,5-dimethyl-1-pyrroline $\mathrm{N}$-oxide by singlet oxygen in aqueous solution. J. Am. Chem. Soc., 118, 1330-1338, 1996.

[33] Matsugo, S., Yan, L.J., Han, D., Tritschler, H.J., and Packer, L.: Elucidation of antioxidant activity of alpha-lipotic acid toward hydroxyl radical. Biochem. Biophys. Res. Commun., 208, 161-167, 1995.

[34] Matsugo, S., Yan, L.J., Han, D., Tritschler, H.J., and Packer, L.: Elucidation of antioxidant activity of dihydrolipoic acid toward hydroxyl radical using a novel hydroxyl radical generator NP-III. Biochem. Mol. Biol. Int., 37, 375-383, 1995.

[35] Smythies, J. and Galzigna, L.: The oxidative metabolism of catecholamines in the brain: a review. Biochim. Biophys. Acta, 10, 159-162, 1998.

[36] Terland, O., Flatmark, T., Tangerås, A., and Grønberg, M.: Dopamine oxidation generates an oxidative stress mediated by dopamine semiquinone and unrelated to reactive oxygen species. J. Mol. Cell. Cardiol., 29, 1731-1738, 1997.

[37] Terland, O., Almås, B., Flatmark, T., Andersson, K.K., and Sørlie, M.: One-electron oxidation of catecholamines generates free radicals with an in vitro toxicity correlating with their lifetime. Free Radic. Biol. Med., 41, 1266-1271, 2006.

[38] Hattoria, N., Wanga, M., Taka, H., Fujimura, T., Yoritaka, A., Kubo, S., and Mochizuki, H.: Toxic effects of dopamine metabolism in Parkinson's disease. Parkinsonism Relat. Disord., 15, S35-38, 2009.

[39] Miyazaki, I. and Asanuma, M.: Dopaminergic neuronspecific oxidative stress caused by dopamine itself. Acta Med. Okayama, 62, 141-150, 2008.

[40] Stokes, A.H., Hastings, T.G., and Vrana, K.E.: Cytotoxic and genotoxic potential of dopamine. J. Neurosci. Res., 15, 659665, 1999. 